\title{
Virtual Reality Technology and Application in Distance Education Presentation
}

\author{
Hua Hong-Xuan \\ Department of Information Science and Engineering, Zaozhuang University, Zaozhuang, Shandong \\ 277160, China \\ hxuanh@163.com
}

Keywords: large virtual reality technology; distance education demonstration; wavelet transform

\begin{abstract}
The development of distance education has important significance for China's education level ascension. Traditional algorithm is used in remote education demonstration, it cannot avoid the disadvantages such as communication channel bandwidth limiting and outside interference, it has poor two-dimensional video demonstration effect. A large scale distance education demonstration method is proposed based on virtual reality technology. Combining virtual reality technology and modern remote education, remote education application environment is constructed based on Shout3D modeling technology. In the transmission process of distance education demonstration data, wavelet transform is used to eliminate the interference, so as to achieve the ideal teaching effect. The experimental results show that, this method is applied in remote education demonstration, it can improve the clarity and satisfactory of images.
\end{abstract}

\section{Introduction}

The development of modern distance education has become a strategic measure of our c ountry for education development. At present, the modern distance education in our country has some problems, mainly include: the traditional Webpage remote education platform on plane animated text description, it is simple, and it is unable to arouse students' learning in terest and enthusiasm. Because the separation of time and space, it cannot provide the learn ing environment of be personally on the scene, and it is unable to realize the emotional co mmunication between the teaching researchers and scholars, the method greatly reduces the initiative learning, it is unable to provide real experimental teaching environment[1-3]. The $t$ raditional method just provides demonstration teaching through the video, leads to the exper iment teaching result is bad, directly affects the cultivation learners' practical ability. Moder $\mathrm{n}$ distance education is the development trend of network with intelligence and virtualization. Virtual reality technology has multi sensory, immersion, interaction and imagination and ot her characteristics, it has the formidable superiority and potential in distance education. The main application of virtual reality technology in distance education include a virtual learning community, simulation laboratory and digital teaching places, and then construct a distance education platform based on virtual reality technology[4,5].

The characteristics of modern distance education make it has been obtained more and $\mathrm{m}$ ore attentions from learners. It uses computer technology, multimedia technology, communic ation technology and network technology as a means of teaching support. This interactive $n$ etwork education can simulate the classroom scene, teaching equipment, experimental scenari os in reality, it can show the vivid simulation. And it presents the vivid scenarios with the combination of image, text, animation, audio and video. Combined with the modern remote education and virtual reality technology, the modern remote education can be promoted.

Virtual reality it developed is based on the computer technology, the core is computer te chnology, human-computer interface technology, sensor technology, simulation technology an $\mathrm{d}$ other technology are combined comprehensively. Three-dimensional space environment is $\mathrm{s}$ imulated for generating realistic space. The application of virtual reality technology can mak e people obtain the scene feeling personally, and has the sense of immersion and interactivi 
ty. Virtual reality technology is used to build immersive virtual reality system, according to different function, it can be classified as simple type of virtual reality system and sharedvi rtual reality system. Virtual Reality Modeling Language (VRML) is the key technology to $r$ ealize virtual reality on the internet. It characterize the 3D world and accelerate the speed of network transmission, reduce the amount of data transmission, the remote education brea ks through the limitation of network bandwidth. Advanced user interface is provided by VR ML, it can make learning object in a natural way and in a virtual environment, it can inter act and influence each other, resulting in the real environment to feel and experience. Virtu al reality modeling language can realize remote real-time transmission and two-way commun ication in education.

\section{Realization of virtual reality technology in Distance Education}

\section{A Construction of distance education application environment}

A prerequisite for the process of constructing a virtual world is to build a three-dimensional model of describing the world, the traditional means taken 3D graphics design software for implementation, such as 3DMAX, MAYA, SoftImage, LightWave3D etc. The traditional modeling method is designed based on computer graphics, sing Polygon or NURBS (non uniform rational B spline Non-Uniform Ration B-Splines) to describe the geometry in 3D scene. The virtual world of traditional modeling obtain spatial forms, perspective transformation free, it has advantages of strong interactive ability, but also has the complexity of the modeling process, the design cycle is long, implementation cost is large, the disadvantages cannot be avoided.

Another kind of 3D model construction technique is taken based on three-dimensional image modeling (IBR), through different angles, get the real scene several. Several photographs are obtained in the perspective and photogrammetry principle, key control points are marked and posited on the object, and thus generate two-dimensional perturbation environment or object representation, three dimensional model framework is enhanced. This kind of commercial software is MetaCreations, RealViz of company ImageModeler Canoma. The advantages of IBR modeling are available to get the $3 \mathrm{D}$ scene by a camera, without need for geometric modeling. The calculation ability of computer requirements is not high, the three-dimensional entity is good, and realistic generation is strong. Its disadvantage is that the virtual world interaction is not enough, form this point of view, the direction of motion of the user is restricted, mutation phenomenon is caused by the switching process between the different viewpoint. Shout3D is a Web3D technology, it inherits the VRML language specification, standards, and reconstruction of the structure using Java. With the help of Java language, Shout3D has the advantages of cross platform, it is easy to transplant and has fast speed, no need of special browser plug-ins and interactive ability. It effectively makes up the deficiency of VRML language. Because of the application of virtual reality remote education demonstration, the process is used by VRML language implementation, so Shout3D technology is adopted to perform the transformation, and the upgrade has practical value. Shout3D adopts the design steps and VRML, virtual campus of Shout3D scenes (including object, animation and so on) is realized through the three-dimensional modeling software (currently the main support of 3DMAX) design, in order to simplify the model file size, try to use the map to establish a complex model to optimize the process, distance education demonstration is pictured on export, The model file is derived for the S3D extension, and it can edit files. The node structure and syntax specification which is consistent with VRML, many new nodes are added to provide higher quality image rendering, animation and interactive function is obtained, S3D scene file is edited, ActiveX is used

to control the form of embedding in the web pages, which can be achieved through the Shout3Dwizard program

\section{B Realization of long-distance education demonstration}

Virtual reality technology is used for image modeling of remote education demonstration, need for remote transmission of $3 \mathrm{D}$ images, before the transmission, it needs to demonstrat e image compression processing, the specific methods are shown as follows: 
The number of image of the remote education demonstration is set as $N$, the transmissi on distance is $l$,the initial moment of image is $t_{0}$, the time of image transmit to the termi nal is $t_{1}$, the pixel gray values of each picture $R_{i}$ is $M$, pixel gray values is $S_{i}$, the followi ng formula is used for image data compression processing of distance education demonstrati on:

$$
\delta_{i}=\frac{|N-M| \sqrt{t_{1}-t_{0}}}{l \times S_{i} \times R_{i}}
$$

The results of the formula can describe the distance education demonstration image comp ression degree. Use the following formula for distance education image wavelet decompositi on transform:

$$
g(y)=\frac{1}{2}\left[X_{\gamma}(0,0) \gamma_{0,0}(y)+X_{\gamma}(0,0) \varphi_{0,0}(y)+X_{\varphi}(1,0) \varphi_{1,0}(y)+X_{\varphi}(1,1) \varphi_{1,1}(y)\right]
$$

Using the above formula, the remote education demonstration is processed for image den oising, eliminate the noise in the image, thus improving the quality of distance education $\mathrm{d}$ emonstration image. Use the following formula to calculate resolution parameters, the distan ce education demonstration image terminal is obtained:

$$
\eta_{i}=\frac{\delta_{i} \times\left(M^{2}-N^{2}\right)}{S_{i}+R_{i}}
$$

According to the method described above, the distance education demonstration images ar e compressed, filtering processing method is used for wavelet transform in the transmission process, the elimination of the interference signal is obtained, so as to get a clear image of the remote education demonstration.

\section{Experimental results and analysis}

In order to verify the effectiveness of improved algorithms, need for an experiment. In $t$ he process of the experiment, the MATLAB simulation software is used to build a remote education demonstration experiment, remote education demonstration image samples are set as 1000 different images, traditional algorithm and the improved algorithm are used for co mparison. Distance education demonstration image transmission results of different algorithm $\mathrm{s}$ are shown in Figure 1.

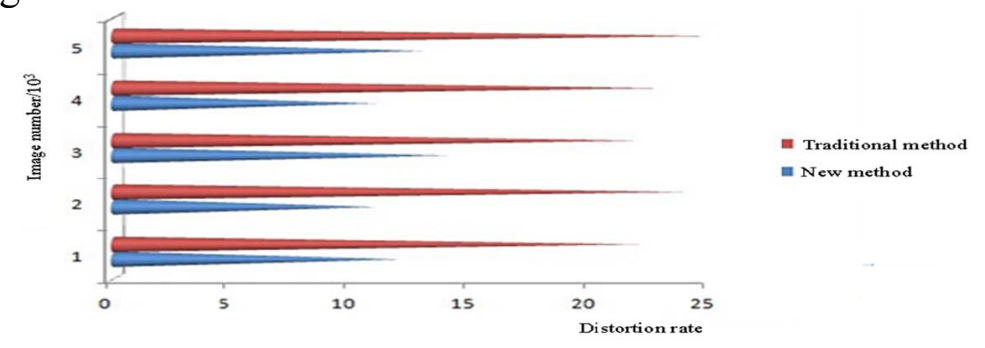

Figure 1.Distortion rate comparison results of this algorithm and the traditional algorithm

According to the results of the experiment, it can be learned that the distance education demonstration image algorithm can improve the distortion rate performance, and the distortion rate is far lower than traditional algorithm. This is because the improved algorithm can make 3D modeling be compressed by virtual reality technology, compression is preferred in the transmission process of $3 \mathrm{D}$ images, and it can eliminate the interference by wavelet transform, thus improve the quality of the 3D image.

The image matching error of education demonstration can be calculated using the following formula 


$$
Q=\frac{\ln [w(W, \hat{W}) / \hat{W}(x, y)]}{\sin [W(x, y)]}
$$

Use different algorithms, the relevant data of distance education demonstration images are expressed in Table 1

Table 1 Comparison of matching results for images presentation of different algorithms

\begin{tabular}{lllll}
\hline & \multicolumn{2}{l}{ Traditional algorithm } & \multicolumn{2}{l}{ Improved algorithm } \\
\cline { 2 - 5 } Image matching methods & $\begin{array}{l}\text { Error } \\
\text { matching } \\
\text { points / total } \\
\text { number } \\
\text { pixels }\end{array}$ & $\begin{array}{l}\text { Matching } \\
(\%)\end{array}$ & $\begin{array}{l}\text { Error matching } \\
\text { points / total } \\
\text { number } \\
\text { pixels }\end{array}$ & $\begin{array}{l}\text { Matching error } \\
(\%)\end{array}$ \\
\hline clockwise rotation 15 degree & $5 / 1000$ & $0.5 \%$ & $0 / 1000$ & $0 \%$ \\
clockwise rotation 30 degree & $19 / 1000$ & $1.9 \%$ & $1 / 1000$ & $0.1 \%$ \\
clockwise rotation 40 degree & $28 / 1000$ & $2.8 \%$ & $8 / 1000$ & $0.8 \%$ \\
Reduce 2 times & $49 / 1000$ & $4.9 \%$ & $18 / 1000$ & $1.8 \%$ \\
Enlarge 2 times & $57 / 1000$ & $5.7 \%$ & $23 / 1000$ & $2.3 \%$ \\
Total & $120 / 3500$ & $12 \%$ & $47 / 1000$ & $4.7 \%$ \\
\hline
\end{tabular}

According to the result of the experiment table as above, we can learn the new method is used in distance education image transmission, the image quality is far higher than the traditional algorithm, satisfactory results are obtained, it has better performance.

\section{Conclusions}

Traditional algorithm is applied in remote education demonstration, it cannot avoid the disadvantages such as communication channel bandwidth limiting and outside interference, it has poor two-dimensional video demonstration effect. A large scale distance education demonstration method is proposed based on virtual reality technology. Combining virtual reality technology and modern remote education, remote education application environment is constructed based on Shout3D modeling technology. In the transmission process of distance education demonstration data, wavelet transform is used to eliminate the interference, so as to achieve the ideal teaching effect. The experimental results show that, this method is applied in remote education demonstration, it can improve the clarity and satisfactory of images.

\section{References}

[1] ZHANG Ren-shang. Network Intrusion Detection System Based on Expert System and Neural Network[J]. Computer Simulation. 2012; 29(9): 162-165.

[2] Dong Yan, Zhu Yongsheng, Liu Cong. Application of Image Fusion Technology in License Plate Recognition[J]. Computer Measurement \& Control. 2013; 21(3): 791-793.

[3] YANG Zhi-Ming, WANG Xiao-Rong, PENG Jun. The Application of BP Neural Networks in Image Segmentation[J]. Computer Science. 2007; 30(3): 234-236.

[4] Zheng Baodao, Wang Huaijie. Design of Remote Image Alarming System Based on ARM/GPRS. Computer Measurement \& Control. 2013; 21(1): 149-151, 159.

[5] Zhao Juan, Zhao Qiang, Wu Fenxia. Self-organizing Map Neural Network Based Image Retrieval Algorithm[J]. Bulletin of Science and Technology. 2013; 29(2): 55-57. 